\title{
Simulation and Detection Performance Evaluation of a UAV-mounted Passive Radar
}

\author{
Evgenii Vinogradov ${ }^{1}$, Dmitry A. Kovalev ${ }^{2}$, Sofie Pollin ${ }^{1}$ \\ ${ }^{1}$ Department of Electrical Engineering, KU Leuven, Belgium \\ ${ }^{2}$ ELEN-ICTEAM, Université catholique de Louvain, Belgium
}

\begin{abstract}
This paper presents the concept of an UAVmounted passive radar. Since the radar has no active transmitter and uses signals transmitted by illuminators of opportunity (IOO), it is a low cost, lightweight, low-power consuming solution perfectly fitting for mobile applications, especially for mounting on a UAV. Moreover, it does not require supplemental frequency allocation and creates no additional interference to existing wireless networks. Longterm evolution (LTE) is a good candidate for illuminators of opportunity (IOO) due to the fact that orthogonal frequency division multiplexing (OFDM) signals are used. Moreover, LTE base stations are widely deployed. In this paper, the detection performance of a drone-mounted passive radar is presented, with various settings in terms of targets, wireless propagation, realistic antenna patterns and signal processing.
\end{abstract}

Index Terms-Passive Coherent Location, Unmanned aerial vehicles, Drones, Radio frequency, Radar, Surveillance, Sensors, LTE

\section{INTRODUCTION}

A sharp rise in the adoption of UAVs (Unmanned Aerial Vehicles) for leisure and professional applications has resulted in personal and government concerns regarding the safety of those systems and even possible aerial attacks. Various commercial establishments and public safety departments worldwide are increasingly deploying counter-UAV measures to address the evergrowing need for security. It has been shown in [1], that so-called surveillance drones (i.e. UAV-mounted systems) can be a more efficient and flexible solution for this purpose then ground-based counterparts.

Detection systems may involve several technologies to detect UAVs, including visual techniques [2], radars [3], analysis of acoustic [4] and electromagnetic emission from onboard radios (e.g. Wi-Fi) [1]. All these techniques (apart from an active radar) satisfy the requirements for UAV-mounted equipment (size, weight, energy efficiency). Unfortunately, visual and sound technologies are limited in range. Electromagnetic emission analysis systems allow detecting distant targets, but a malicious UAV most probably will be autonomous (intentionally using a "silent-mode").

Passive radars [5] as well receive electromagnetic radiation present in the environment, but the main difference is that they analyze the changes in a signal radiated from an external transmitter to detect various targets. This makes them good candidates for various aerial applications such as, for example, malicious drone detection for big events or security sensitive areas.

In this paper we present a UAV-mounted passive radar system for intruder UAVs detection. The first case study considers an event scenario where the UAV equipped with the detection system benefits from a good view of the no-fly zone and from suppression of the direct channel due to the proposed geometry. Next, for the scalable solution, the limitations introduced by the propagation conditions in a realistic sub-urban environment are identified and analyzed.

The main contributions of this work can be summarized as follows:

- An overview and an analysis of the passive radar system parameters and the signal processing suitable for an UAV-mounted solution

- Simulation based target detection performance evaluation for specific (open space no-fly zone) and more general scenarios assuming realistic antenna patterns and 3D map of a real city

- A characterization of the realistic suburban environment by altitude and distance dependence of the line-of-sight probability

- An overview of potential issues as well as potential research directions within the subject in the future

\section{Passive Coherent Location}

A Passive Coherent Location system (PCL) is a passive radar that does not have its own source of the radar signal and exploits emissions from so-called illuminators of opportunity (IOO) to detect objects [5]. A PCL radar has two receiving channels denoted as a reference channel and a surveillance channel. The channel between the radar receiver and IOO provides a reference signal. The surveillance channel contains all the copies of the signal emitted by IOO and reflected by static and dynamic objects. Note, that all the nodes (IOO, target and radar) must be in line-of-sight (LOS) for the reliable detection. The reference signal is used to perform matched filtering of the surveillance signal in order to extract targets echoes.

In this paper, we investigate a possibility of building an airborne passive radar with one omnidirectional 
antenna so that the weight and complexity of the final system could be acceptable for the aerial surveillance system mounted on a small UAV. The choice of the IOO and the specifics of the targets that should be detected by such a system are described hereafter.

\section{A. Illuminators of opportunity}

Various IOO have been used for PCL. The most recently used sources are:

- Global Navigation Satellite Systems (GNSS) [6]

- Wi-Fi [7]

- Digital Video Broadcasting-Terrestrial (DVB-T(2)) [8], [9]

- Global System for Mobile communications (GSM) [10] and Long-Term Evolution (LTE) [11], [12],

GNSS-based techniques have some advantages since the signals are available globally and they do not interfere as in cellular type systems. Theoretical range resolution is about $15 \mathrm{~m}$, but due to the low signal strength, the detected target should have large radar cross-section (RCS) (about $50 \mathrm{~m}^{2}$ [6]), which is not the case for a typical UAV. Wi-Fi transmitters cannot be used since the transmit power is low. DVB-T(2) transmitters emit high power that can provide a good coverage. In general, DVB-T(2) is a good candidate for the IOO, however, the range resolution is smaller compared to LTE signals [13]. Globally, GSM coverage is still larger than LTE, but the signal bandwidth in GSM systems is about $200 \mathrm{kHz}$, which provides approximately $1.5 \mathrm{~km}$ range resolution [13].

The LTE signal has properties that make it a good candidate for an illuminator of opportunity for passive radar systems: (1) Frequency bands ranging from $800 \mathrm{~Hz}$ to $3500 \mathrm{MHz}$; (2) OFDM structure of the signal guarantees low sidelobes of the ambiguity function; (3) Bandwidth up to $20 \mathrm{MHz}$. Two LTE bands are the most used in Europe: Band 3 (downlink: 1805 - $1880 \mathrm{MHz}$ ) and Band 20 (downlink: 791 - $821 \mathrm{MHz}$ ). The results in [13] show that the LTE signal can provide a good range resolution of 7.5-8.6 m. The expected Doppler resolution is 0.17 and $0.33 \mathrm{~m} / \mathrm{s}$, respectively. In this work, the LTE signal is used to analyze the characteristic of the considered system.

\section{B. Potential targets}

The main objective of the analyzed system is to perform detection of the potential micro-UAV intruders. A fundamental issue limiting the detection of such objects is their low RCS.

RCS is a measure of the amount of electromagnetic energy that is reflected from the object. Different factors influence the value of the RCS: i) material of which the target is made of; ii) the shape and size of the target (absolute and in relation to the wavelength of the radar signal); iii) angles of the incident and reflected electromagnetic waves.

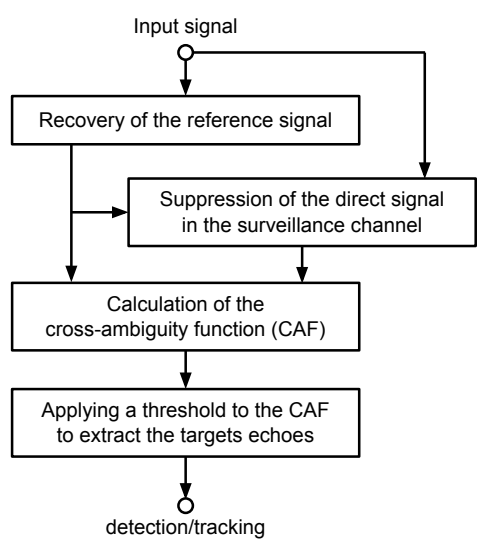

Fig. 1. Flow-diagram of the signal processing in PCL

The interest in UAV technologies and the widespread safety and security concerns regarding UAV usage resulted in several papers dedicated to RCS measurements. LTE frequencies were used in [14] for measuring the signatures of a static UAV. It was reported that for DJI Phantom 3, the RCS varies depending on the orientation and movement from -23 to $-10.43 \mathrm{dBm}^{2}$ (0.005-0.091 $\mathrm{m}^{2}$ ). Some other target types can be found in [15]. The low RCS of the potential targets define the specifics of the signal processing of the received signals. This is described in the following section.

\section{SignAL PROCESSING}

To extract the information about the target (its position and velocity), the received signal is processed as shown in Fig. 1. In this work, it is considered that only one receiving channel is available. No tracking is performed after detection of targets.

\section{A. Recovery of the reference signal}

Remodulation of the signal is performed for two reasons. First, due to the presence of pilot carriers in the spectrum of the OFDM signal, the cross-ambiguity function (CAF) contains deterministic peaks (also called unambiguous peaks) scattered over the delay-Doppler shift plane. An example of the ambiguity function of the LTE signal is shown in Fig. 2. The peaks generated by pilots are clearly seen at nonzero Doppler frequency shifts. They can complicate target echoes detection and cause false alarms. To mitigate the influence of the pilot peaks in the OFDM signal, the amplitude of the pilot subcarriers should be reduced in the recovered signal [16].

Second, the interfering direct signal should be suppressed from the surveillance channel to enable the detection of the weak targets echoes [17]. For that aim, it is needed to obtain a clean copy of the reference signal without any delayed components that can affect the filtering process. To obtain the reference signal, it 


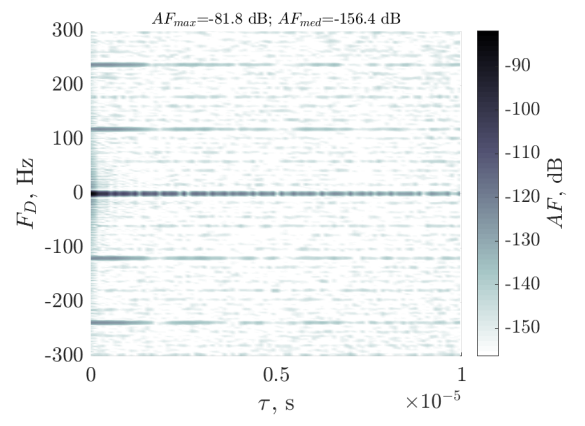

Fig. 2. Ambiguity function of the LTE signal

should be recovered (remodulated) from the surveillance channel. Remodulation of the OFDM-like reference signal consists of the following main steps: first, a time synchronization is performed to find the beginning of each OFDM symbol in time; then, frequency synchronization is done to align all OFDM subcarriers at the centers of the proper FFT bins; finally, pilot subcarriers are used for channel equalization and OFDM QAM symbol constellations are decoded using soft/hard decoding. Then, the procedure is executed in the reverse order to obtain a clean reference signal template.

Remodulation of the reference signal is out of the scope of this work. In the following simulations, it is assumed that the reference signal is directly available. It is shown in [18] that the quality of the reference signal recovery significantly affects the detection range of the radar. The presence of the unambiguous peaks in the $\mathrm{CAF}$ is ignored. To avoid the influence of those peaks on the estimation of the system performance, locations of the targets are chosen so as to avoid their intersection with the position of the peaks.

\section{B. Suppression of the interfering direct signal}

Target echoes can be masked by the direct signal and its delayed copies as well as by the reflections from other targets with a high RCS. Adaptive filters are used to suppress those masking signals. Selection of the filter is a compromise between the degree of suppression, the time needed for the filter to converge, and the computational cost. In general, adaptive filtering can suppress the interference signal by 50-65 dB [17], [19]. To increase the Doppler resolution and the SNR, a longer coherent integration time is used (See Section III-C). Longer duration of the processed signal increases the computational costs of adaptive filtering. To reduce the time needed to process the signal, the reference and surveillance signals can be divided into overlapping intervals. Adaptive filtering is then applied in parallel to each of the intervals. In this work, the NLMS filter is used to suppress the strong direct signal in the surveillance channel. The influence of the delayed copies of the direct signal and strong echoes from other moving targets are omitted in the current work to simplify the simulations.

\section{Cross-ambiguity function}

The CAF is calculated between the recovered reference and filtered surveillance signals to determine the bistatic locations and bistatic Doppler shifts of the target echoes

$$
\begin{aligned}
& \left|\chi\left(\tau, f_{D}\right)\right|= \\
& \left|\int_{-\infty}^{+\infty} s_{\text {ref }}(t) \quad s_{s r v}^{*}(t-\tau) \exp \left(j 2 \pi f_{D} t\right) d t\right|,
\end{aligned}
$$

where $s_{\text {ref }}(t)$ is the recovered reference signal, $s_{\text {srv }}(t)$ is the surveillance signal after adaptive filtering, $\tau$ is the time delay and $f_{D}$ is the Doppler frequency shift. This matched filtering of the surveillance signal (or pseudomatched if the reference signal is modified) provides processing gain $G_{s p}=T \cdot B$ [5], where $B$ is the effective receiver bandwidth and $T$ is the coherent integration time, which depends on the time for which the target echoes remain coherent.

\section{Target detection}

After the CAF is calculated, a threshold has to be determined to perform a target detection. In order to maintain a constant probability of false alarm $\left(P_{f a}\right)$ the detection threshold is adapted according to an estimate of the noise variance. A cell-averaging constant false alarm rate (CA-CFAR) detector with a guarded cells algorithm [20] is used in this work. A cell corresponds to a given delay and Doppler shift coordinates of the CAF. The threshold level is calculated by estimating the level of the noise floor in training cells around the cell under test (CUT). This can be found by taking a block of cells around the CUT and calculating the average power level. Target returns may spread across multiple cells. To avoid the influence of this phenomenon on the noise estimation, cells adjacent to CUT (guard cells) are ignored. A target is declared present in the CUT if the $\mathrm{CAF}$ at a given cell is both greater than in all adjacent cells and greater than the local average power level.

The algorithm operates on the full CAF surface. The optimum parameters were found empirically: the number of training and guard cells are 10 and 4, respectively.

\section{SURVEILlANCE DRONE FOR PUBLIC EVENTS}

The first case study considers a UAV-mounted passive radar for intruder drone detection during big-scale public events (e.g. music festivals). Often, mobile operators use mobile or temporary LTE base stations $(\mathrm{eNb})$ for such events to provide service to their customers. We suggest to use such an $\mathrm{eNb}$ as an IOO for passive coherent location: base stations have 3 sectoral antennas; the antenna height is usually lower than in a typical installation (up to $10-15 \mathrm{~m}$ ); the emitted power varies between 23 and $46 \mathrm{dBm}$. 

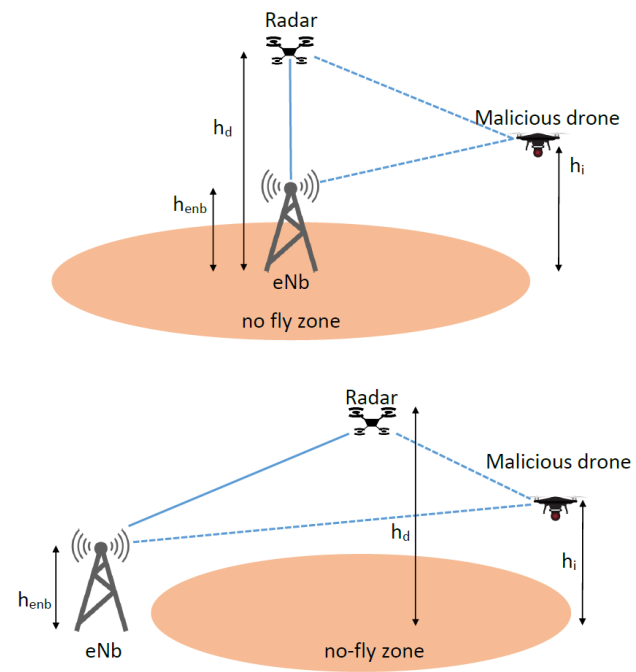

Fig. 3. UAV-mounted passive radar for intruder drone detection top: during public events hovering above an eNb; bottom: scalable surveillance solution
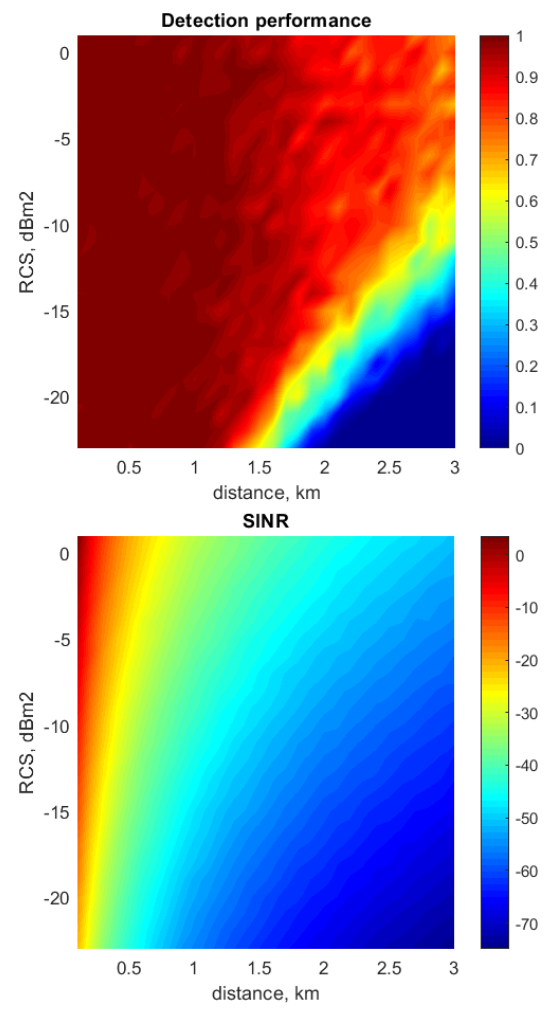

Fig. 4. Detection performance and SINR levels as a function of the target RCS and distance from the receiver for $P_{f a}=10^{-3}$
In this section, a surveillance UAV hovering right above the base station (see Fig. 3) is analyzed. Table I contains the parameters used for numerical simulations. Three sectoral Kathrein 734314 and dipole antennas are used at the transmitter and receiver side, respectively. Free-space path loss propagation conditions are assumed.

TABLE I

PARAMETERS OF THE SIMULATION.

\begin{tabular}{lc}
\hline \hline Parameter name & Value \\
\hline Carrier frequency & $1800 \mathrm{MHz}$ \\
Transmit power & $46 \mathrm{dBm}$ \\
$h_{e n b}$ & $10 \mathrm{~m}$ \\
Tx antenna gain & $18,14 \mathrm{dBi}$ \\
Tx horizontal beamwidth (at $3 \mathrm{~dB})$ & $65^{\circ}$ \\
Tx vertical beamwidth $($ at $3 \mathrm{~dB})$ & $8^{\circ}$ \\
Rx antenna gain & $2,04 \mathrm{dBi}$ \\
Receiver noise figure & $6 \mathrm{~dB}$ \\
\hline
\end{tabular}

By using the considered geometry, the radar benefits from significant suppression of the direct channel (solid line) due to the minima of the antenna patterns: the difference between the $\mathrm{Tx}$ and $\mathrm{Rx}$ antenna gains for the direct channel and surveillance channel (eNb-targetradar, dashed line) can be as high as $50 \mathrm{~dB}$. Since the distance between eNb and the UAV is $80 \mathrm{~m}$, the propagation attenuation is about $75 \mathrm{~dB}$, which leads to $\mathrm{SNR} \geq 20 \mathrm{~dB}$ for the direct channel. This level supports the assumption that the reference signal can be recovered from the same omnidirectional antenna as used for the surveillance channel.

The aim of the simulation is to estimate the target detection probability depending on its RCS and the distance between the radar and the target. For that, various positions of the malicious drones are simulated for relatively high altitude, $h_{i}=90 \mathrm{~m}$. These parameters represent a worst-case scenario since the targets are out of the main lobe of the eNb antenna pattern. This means that the received echoes are weaker than for lower target positions. Each simulation run includes 4 UAVs with identical RCS. The simulated RCS is ranging from $-23 \mathrm{dBm}^{2}$ (corresponding to the smallest UAV RCS value found in the literature) to $1 \mathrm{dBm}^{2}$ (corresponding to a large UAV). In total, 25 RCS values are used, and for each RCS value, 4000 random target locations in a circle with $3 \mathrm{~km}$ radium are simulated. Targets are moving in arbitrary directions in the $x y$-plane with velocities drawn from a uniform distribution (maximum $15 \mathrm{~m} / \mathrm{s}$ ).

The received signal (a mixture of the reference and 4 echo signals) is processed as it is described in Section III. The coherence integration interval of $0.2 \mathrm{~s}$ is used for CAF calculation resulting in a processing gain of $G_{s p}=66 \mathrm{~dB}$. The probability of false alarm $P_{f a}$ used in the CA-CFAR detector is $10^{-3}$. After the CFAR 

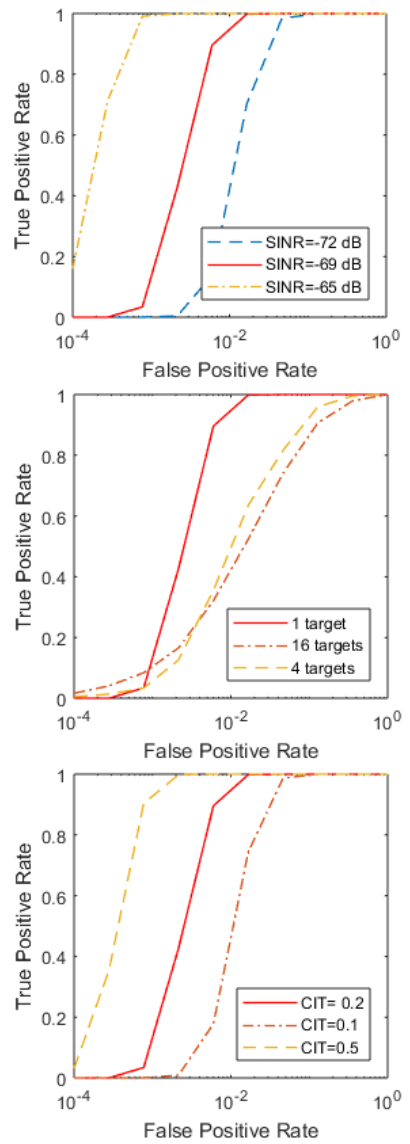

Fig. 5. Receiver operating characteristic. top: for different SINR (CIT $=0.2 \mathrm{~s}$ ); middle: for different number of targets per simulation run $(\mathrm{CIT}=0.2 \mathrm{~s})$; bottom: for different coherent integration times

processing, the extracted delays and Doppler shifts of each target are compared with the ones that were used at the input of the simulator to determine if the target was detected or not.

Fig. 4 presents results of the simulation. The UAVs with smallest considered RCS, $\sigma=-23 \mathrm{dBm}^{2}$, are detected with a probability higher than 0.95 within a distance of $1 \mathrm{~km}$ from the radar. The signal-to-interferenceplus-noise ratio (SINR) level corresponding to the targets detected with this probability is higher than $-55 \mathrm{~dB}$ but by using an adaptive filter to suppress the direct interference signal, as described in Section III, a good detection performance can be achieved.

Fig. 5 presents the receiver operating characteristic (ROC) curves. For this set of simulations, the targets are located in the direction of the maximum antenna gain (in azimuth) available at altitude $h_{i}=90 \mathrm{~m}$ for correspondent distance. Each target is moving in an arbitrary direction with the random speed (maximum $15 \mathrm{~m} / \mathrm{s}$ ). The inspected SINR levels (Fig. 5, top) correspond to targets (one per simulation run) located at distances $d=3,2.5$ and $2 \mathrm{~km}$. The higher detection probability than that shown in Fig. 4 is caused by a
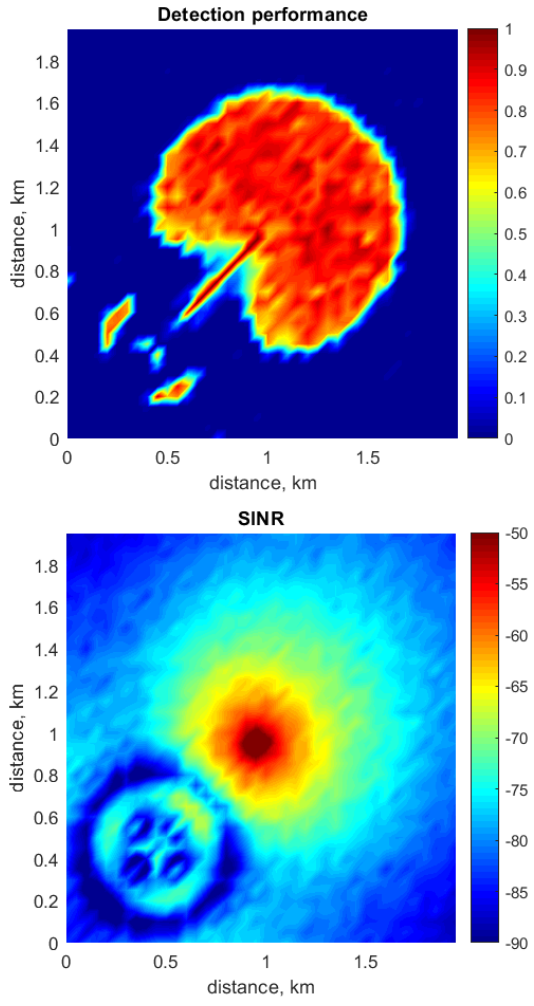

Fig. 6. Detection performance $\left(P_{f a}=10^{-3}\right)$ and SINR levels as a function of distance for the scalable solution

higher antenna gain for the chosen direction.

In Fig. 5 (middle), the ROC curves are shown for 16, 4 and 1 targets $(d=2.5 \mathrm{~km})$. Presence of more intruders affects the detection performance. Fig. 5 (bottom) demonstrates an improvement of the detection performance with increasing of the CIT.

These results show that the proposed solution can be effective for monitoring of the no-fly zones.

\section{Scalable Surveillance Drone Solution}

In the previous section, a surveillance solution for a specific yet relevant scenario was discussed. However, it is desirable to have a scalable solution (see Fig. 3) that can be deployed for a wide range of deployment environments (rural, suburban and urban) without the need of an extra eNB or the constraint that the UAV should hover above the eNB. One of the main advantages of the UAVmounted passive radar in comparison to a ground-based radar is that it provides better propagation conditions at high altitudes (i.e. higher probability of having line-ofsight in the reference and surveillance channels).

This section identifies the limitations of the previously described solution in the case of the deployment in any location in a realistic suburban environment.

\section{A. Direct signal interference}

The first set of simulations demonstrates the influence of the reference signal interference on the target detec- 
tion probability. For a fair comparison, the transmitter and receiver (i.e. power, antenna patterns, noise figure) are identical to the ones used in Section IV. The aerial radar is located at $700 \mathrm{~m}$ distance from the IOO. The antenna height, $h_{e n b}=20 \mathrm{~m}$, corresponds to a typical LTE deployment in a sub-urban scenario. The radar and target heights are $h_{d}=h_{i}=90 \mathrm{~m}$; the radar remains at the same position during the surveillance period. Targets with RCS, $\sigma=-23 \mathrm{dBm}^{2}$, (4 targets per simulation run) are randomly placed on a grid around the radar. The number of simulation runs equals to 16000 .

In this geometry, the radar does not benefit from the reference signal attenuation due to the antenna patterns. Instead, the total antenna gain in the reference channel is around $16 \mathrm{~dB}$. This causes significantly lower SINR levels: up to $-66 \mathrm{~dB}$ in the area of consideration due to the antennas influence, which is partly compensated by higher path loss of about $90 \mathrm{~dB}$. Higher direct signal interference causes high side lobes of the direct signal autocorrelation in the CAF, that mask weak target echoes.

Fig. 6 shows the simulation results. The location of the radar corresponds to the middle of the figure; the eNb has coordinates $[500 \mathrm{~m}, 500 \mathrm{~m}]$. The simulation confirms that this geometry causes a significant drop in SINR levels and, consequently, decreasing of the detection probability. SINR variations around the eNb are caused by the side-lobes of the eNb antenna pattern. The detection probability is low in the area between the $\mathrm{eNb}$ and the radar due to the low SINR. Relatively to the setup considered in Section IV, the used geometry provides target detection within smaller areas of 500$700 \mathrm{~m}$ from the radar (depending on the direction) with 0.75 probability of detection.

These results are obtained only for the targets located at the altitude of $90 \mathrm{~m}$. In the simulation, the radar and targets receive the signal from a side-lobe of the eNb antenna pattern. For lower flying targets (i.e. closer to the main lobe), SINR and hence the detection probability are higher. The following subsection develops the influence of the suburban environment on the detection characteristics.

\section{B. Suburban environment influence}

In the previous subsection, it is shown how the change in SINR levels affects the detection probability for the scalable scenario. Another important factor that must be considered in a realistic environment is LOS conditions on all the links shown in Fig. 3: absence of LOS on any of the channels makes the detection impossible and causes further degradation of the system performance. This section inspects the influence of the suburban environment on the LOS conditions. For this purpose, a 3D map of Heverlee, Belgium is used and the real positions of two existing eNbs are considered.
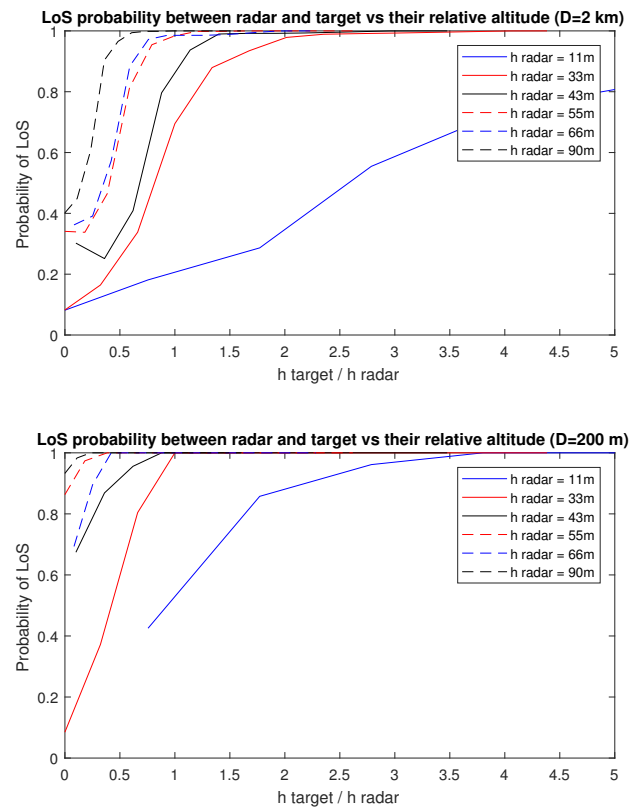

Fig. 7. Altitude dependence of the LOS probability against relative altitudes of the radar and the target

Minimum height of the radar, $h_{d, \min }(i)=$ $\{11,33,43,33,55,66\} \mathrm{m}$, at each distance from the serving eNb $L_{1(2), i}=\{245,495,740,985,1230\} \mathrm{m}$ is found as the minimum altitude (in respect to the groundlevel) where a LOS link between the correspondent $\mathrm{eNb}$ and the receiver exists. The LOS area of an eNb is mostly defined by the surrounding environment: namely by the highest buildings in the direction of the link. The maximum height of the radar $h_{d, \max }$ is set to $90 \mathrm{~m}$ above the ground level. The minimum height of the target corresponds to the lowest ground level of the considered area. The maximum height of the target $h_{i, \max }$ is $180 \mathrm{~m}$ above the minimum target height.

To describe the environment, it is proposed to use a probability of having the LOS between two points in the environment. Fig. 7 shows an influence of the radar and target altitudes on the LOS probability between these two nodes. The targets are located within a cylinder with a diameter of $2 \mathrm{~km}$ centered around the radar position. For the considered landscape, at the altitude $h_{t}=h_{r}=11 \mathrm{~m}$, the probability of LOS is around 0.2 . It is intuitive that the higher the radar altitude is, the easier it is to detect lower targets as LOS conditions exist with a high probability. To have LOS between the radar and the target separated by $2 \mathrm{~km}$ and flying at the same altitude, the nodes have to be located higher than $55 \mathrm{~m}$ (see Fig. 7, top). Consequently, for detection over a large area, the radar should fly at a high altitude: this provides a higher LOS probability and SINR levels due to favorable LOS conditions and lower antenna gains, 
respectively.

In the case detection is needed over a shorter range (only targets located not farther than $100 \mathrm{~m}$ from the radar), LOS probability grows to 0.5 even at $h_{t}=$ $h_{r}=11 \mathrm{~m}$. At all altitudes higher than $33 \mathrm{~m}$, the LOS probability equals 1 for the cylinder with a diameter of $200 \mathrm{~m}$ (see Fig.7, bottom).

Summarizing, to achieve the performance obtained in Section V-A, the airborne passive radar should fly at a high altitude $(\geq 66 \mathrm{~m})$ to ensure LOS conditions between the $\mathrm{eNb}$, the target, and the radar. Lower radar positions result in further degradation of system performance due to: (1) the signal blockage by the environment and (2) higher interference from the reference signal due to the higher transmitter antenna gains.

\section{CONCLUSION}

This paper studies the feasibility of using a UAVmounted surveillance system to detect a range of UAV types. It is shown that this solution can achieve good detection performance for a specific yet practical scenario. In addition, a UAV-mounted radar can be used in a variety of more generic scenarios with good detection performance depending on the UAV and target altitude. A high detection probability $(\geq 0.95)$ of small UAVs is expected for large distances (around $1 \mathrm{~km}$ ) if a scenario when the system located above the IOO is considered. When the radar is arbitrarily placed in a suburban environment, the detection probability significantly deteriorates $(\approx 0.75$ for $500-700 \mathrm{~m}$ distances for high radar altitudes). Further deterioration of the performance is expected if the flying altitude becomes smaller due to lower LOS probability and higher direct signal interference. One possible solution to improve the performance is to use more complex antennas at the receiver to amplify the echo signals and/or to form the antenna pattern nulling in the IOO direction. Next steps in the development of a practical UAV-mounted passive radar are the implementation of: i) clutter mitigation (e.g. moving target indication); ii) target classification (e.g. by means of micro-Doppler signature analysis); iii) target tracking. In combination with other techniques described in [1]-[4], the passive radar can be a part of a reliable aerial system for UAVs detection over a large set of environments. At the next step, it is planned to conduct the measurements in order to (1) establish the limits of operability in the real world scenario; (2) estimate the influence of the real background clutter, especially if the radar is moving; (3) estimate the influence of the quality of the reference signal recovery on the detecion performance; (4) check all the assumptions that were made and specify the parameters of a possible practical realization.

\section{ACKNOWLEDGEMENTS}

This work was supported by the Research Foundation Flanders (FWO), project no. S003817N "OmniDrone”.

\section{REFERENCES}

[1] M. M. Azari, H. Sallouha, A. Chiumento, S. Rajendran, E. Vinogradov, and S. Pollin, "Key technologies and system tradeoffs for detection and localization of amateur drones," IEEE Communications Magazine, vol. 56, no. 1, pp. 51-57, Jan 2018.

[2] A. Rozantsev, S. Sinha, D. Dey, and P. Fua, "Flight Dynamicsbased Recovery of a UAV Trajectory using Ground Cameras," in CVPR17, July 2017.

[3] F. Hoffmann, M. Ritchie, F. Fioranelli, A. Charlish, and H. Griffiths, "Micro-Doppler based detection and tracking of UAVs with multistatic radar," in IEEE RadarConf, May 2016, pp. 1-6.

[4] J. Kim, C. Park, J. Ahn, Y. Ko, J. Park, and J. C. Gallagher, "Real-time UAV sound detection and analysis system," in IEEE Sensors Applications Symposium, March 2017, pp. 1-5.

[5] M. Cherniakov, Bistatic Radars: Emerging Technology. Wiley, 2008.

[6] X. He, T. Zeng, and M. Cherniakov, "Signal detectability in SSBSAR with GNSS non-cooperative transmitter," IEE Proceedings - Radar, Sonar and Navigation, vol. 152, no. 3, pp. 124-132, June 2005.

[7] P. Falcone, F. Colone, and P. Lombardo, "Potentialities and challenges of WiFi-based passive radar," IEEE Aerospace and Electronic Systems Magazine, vol. 27, no. 11, pp. 15-26, Nov 2012.

[8] M. Conti, F. Berizzi, D. Petri, A. Capria, and M. Martorella, "High range resolution DVB-T Passive Radar," in The 7th European Radar Conference, Sept 2010, pp. 109-112.

[9] A. Barkhatov, E. Vorobev, and A. Konovalov, "Experimental results of DVB-T2 passive coherent location radar," in 2017 IEEE Conference of Russian Young Researchers in Electrical and Electronic Engineering, Feb 2017, pp. 1229-1232.

[10] P. Krysik, P. Samczynski, M. Malanowski, L. Maslikowski, and K. S. Kulpa, "Velocity measurement and traffic monitoring using a GSM passive radar demonstrator," IEEE Aerospace and Electronic Systems Magazine, vol. 27, no. 10, pp. 43-51, Oct 2012.

[11] R. Raja Abdullah, A. Salah, and N. Rashid, "Moving target detection by using new LTE-based passive radar," Progress In Electromagnetics Research B, vol. 63, no. 1, pp. 145-160, 2015.

[12] S. Bartoletti, A. Conti, and M. Z. Win, "Passive radar via LTE signals of opportunity," in 2014 IEEE International Conference on Communications Workshops (ICC), June 2014, pp. 181-185.

[13] A. A. Salah, R. S. A. R. Abdullah, A. Ismail, F. Hashim, C. Y. Leow, M. B. Roslee, and N. E. A. Rashid, "Feasibility study of LTE signal as a new illuminators of opportunity for passive radar applications," in 2013 IEEE International RF and Microwave Conference (RFM), Dec 2013, pp. 258-262.

[14] S. Kim, S. J. Lee, J. H. Jung, and Y. Kim, "Radar cross section measurements of quadcopters using long term evolution frequency," in 2017 IEEE Asia Pacific Microwave Conference, Nov 2017, pp. 1294-1297.

[15] R. A.Stonier, "Stealth aircraft \& Technology From World War II to the Gulf. Part II: Applications and Design," SAMPE Journal, vol. 27, no. 5, September/October 1991.

[16] D. A. Kovalev and V. I. Veremyev, "Correction of DVB-T2 signal cross-ambiguity function for passive radar," in 2014 International Radar Conference, Oct 2014, pp. 1-4.

[17] R. Cardinali, F. Colone, C. Ferretti, and P. Lombardo, "Comparison of clutter and multipath cancellation techniques for passive radar," in 2007 IEEE RadarConf, April 2007, pp. 469-474.

[18] G. Fang, J. Yi, X. Wan, Y. Liu, and H. Ke, "Experimental research of multistatic passive radar with a single antenna for drone detection," IEEE Access, pp. 1-1, 2018.

[19] M. Malanowski, "Comparison of Adaptive Methods for Clutter Removal in PCL Radar," in 2006 International Radar Symposium, May 2006, pp. 1-4.

[20] M. Richards, Fundamentals of Radar Signal Processing, ser. Professional Engineering. Mcgraw-hill, 2005. 